\title{
Large-Eddy Simulation of Dispersion from Surface Sources in Arrays of Obstacles
}

\author{
V.B.L. Boppana · Z-T Xie · I.P. Castro
}

Received: date / Accepted: date

\begin{abstract}
Towards meeting the objective of simulating heat transfer processes in urban areas, the study of dispersion from a scalar (ground) surface area source has been addressed as a first step, as dispersion from such a source is in some ways analogous to heat transfer from the surface. Two different urban-like geometries are considered in this study: an array with uniform height cubes and an array with random height cuboids. Some point measurement dispersion experiments in a wind tunnel have previously been carried out in identical arrays using a naphthalene sublimation technique. Large-eddy simulations (LES) of these experiments have been performed as a validation study and the details, presented here, demonstrate the influence of the roughness morphology on the dispersion processes and the power of LES for obtaining physically important scalar turbulent flux information.
\end{abstract}

Keywords Area source · Dispersion · Passive scalar · Urban canopy

\section{Introduction}

In urban climatology, accurate estimation of sensible heat flux is a matter of utmost importance in view of the continuing surge in urbanisation. This quantity forms a core component in the surface energy balance and hence its estimation is crucial in parameterisation of urban areas, for modelling air pollution dispersion in general atmospheric conditions and for improving urban planning and design for human comfort. Numerous computational fluid dynamics (CFD) simulations (mostly with two-dimensional surface morphologies), field campaigns and wind-tunnel modelling studies have been carried out in the past decade or two to understand the impact of thermal effects in and around the urban canyon.

V. B. L. Boppana · Zheng-Tong Xie · Ian P. Castro

School of Engineering Sciences

University of Southampton

Tel: $+44(0) 2380592320$

Fax: +44(0)2380593058

E-mail: V.B.Boppana@soton.ac.uk 
As part of the Nantes'99 experimental campaign, Louka et al. (2002) studied the variation of direct solar heating of the street sides and the ground. At a height of $12 \mathrm{~m}$ from the ground they observed thin thermal layers of no more than $0.2 \mathrm{~m}$ thick on building walls. The most active regions were sometimes only $0.02 \mathrm{~m}$ thick. The street canyon had an aspect ratio $h / w$ ( $h$ being the mean building height and $w$ being the width of the canyon) of 1.4. Due to a lack of flow measurements, the detailed dynamics within these thin layers are not understood but the effect of the temperature differences on the larger-scale flow dynamics within the street canyon was nonetheless found to be negligible. Similar conclusions were drawn by Idczak et al. (2007) from their joint ATREUS-PICADA experiment (ATREUS: Advanced Tools for Rational Energy Use towards Sustainability; PICADA: Photo-catalytic Innovative Coverings Applications for Depollution, Demilecamps and André 2005). The wind tunnel study by Kovar-Panskus et al. (2002) on a square section cavity likewise demonstrated a very weak secondary flow near the ground at very low Froude number (based on the freestream velocity, canyon height and the difference between freestream reference temperature and the heated wall temperature) but found no evidence of large wall heating effects on the canyon flow field. On the other hand, in another set of field studies by Georgakis and Santamouris (2006) on a deep canyon of aspect ratio 3.3, it was observed that for approach wind speeds less than $5 \mathrm{~ms}^{-1}$ the air flow patterns were dominated by thermal phenomena and intermittent vortices at the building corners. They found higher temperature above the canyon when compared to canyon temperature and the maximum difference was found to be around $5^{\circ} \mathrm{C}$. However, within the canyon no temperature stratification with height was found and this was also observed by Offerle et al. (2007) in a street canyon of aspect ratio 2.1. They deduced that this could be due to the primary vortex in the canyon, driven by the strong shear layer at the canyon top, leading to relatively thorough mixing throughout the canyon. Recently, Kanda and Moriizumi (2009) conducted the COSMO (Comprehensive Outdoor Scale MOdel) experiments that comprised various urban-like geometries and the momentum fluxes, sensible heat fluxes and bulk transfer coefficients were examined. They found that the bulk heat transfer coefficient was less sensitive to the surface geometry compared to the bulk momentum transfer coefficient.

The results of these and other studies, showing rather different influences of thermal effects on the flow field within the canopy, can in principle be explained on the basis of the values of the different governing parameters in each case. Amongst the latter must be listed the geometrical parameters governing the layout of the buildings constituting the effective surface roughness, Richardson numbers based on appropriate velocity scales and the temperature differences between the walls and the flow aloft, and flow Reynolds numbers (although if these latter are large enough one might expect only a weak influence). In order to have a deeper understanding of the complex factors that are involved in the transport of heat in urban canyons it would be useful to perform numerical simulations, perhaps for appropriate parametric studies, provided of course that adequate modelling strategies can be found. Such modelling should be verified against the available experimental data, whether from the field or the laboratory. 
Many 2D (two dimensional) and quasi-2D computational studies on such problems have been carried out by various researchers using different CFD approaches. Kim and Baik $(1999,2001)$ examined the thermal effects in a street canyon of various aspect ratios $(0.6 \leq h / w \leq 3.6)$ using a $2 \mathrm{D}$ numerical Reynolds-averaged NavierStokes (RANS) code and $k-\varepsilon$ turbulence closure. In their two separate studies, with uniform heating of windward wall/leeward wall/street bottom (Kim and Baik, 1999) and temperature differences between the streets and the air above the canopy in the range 0 to $16 \mathrm{~K}$ (Kim and Baik, 2001), they observed a significant influence of heat on the flow field. A similar study by Sini et al. (1996) using a 3D numerical code (CHENSI) with a $k-\varepsilon$ turbulence closure scheme on a $2 \mathrm{D}$ canyon of aspect ratio about unity, showed a net increase in vertical exchange rate when the leeward wall or the bottom street was heated and a reverse behaviour with windward wall heating. Louka et al. (2002) imitated their field study using the same numerical code and their numerical results over-predicted the field buoyancy effects. Another 2D study by Solazzo and Britter (2007) using FLUENT and the $k-\varepsilon$ turbulence model on a canyon of aspect ratio varying from 0.5 to 4 showed a spatially uniform distribution of temperature within the canyon when the bounding facets were either uniformly or partially heated, which led them to deduce a constant non-dimensional exchange velocity between the street canyon and the flow above. Cai et al. (2008) used largeeddy simulation (LES) to compute spatial and temporal mean scalar fluxes at the roof level as a function of aspect ratio $(0.33 \leq h / w \leq 2)$ and found good agreement with wind-tunnel data (Barlow and Belcher, 2004).

There also exist simpler models like LUMPS (Local - scale Urban Meteorological Parameterization Scheme), described by Grimmond and Oke (2002), which calculates heat fluxes for the urban environment. However, these authors state that the model may need further improvement to account for the wide variation in urban morphology. On the other hand, Harman et al. (2004) developed such a model, specifically to predict the magnitude and geometric dependence of the vertical flux of a scalar from each facet of a 2D street canyon and the prediction was found to be in good agreement with the wind-tunnel data (Barlow and Belcher, 2004).

Although the above $2 \mathrm{D}$ or quasi-2D studies give useful indications of the heat effects on flow and dispersion in the urban environment, insights for practically important configurations require robust 3D simulations, not least because measurements in the field are quite challenging and very expensive. Very few full 3D CFD studies have been undertaken thus far, owing partly to the complexity of the typical domain and the expense of such computations. Mathey et al. (1999) used LES to study the flow past a single heated cube and compared the surface temperature profiles and heat transfer coefficient with experiments (Meinders and Hanjalić, 1999). They obtained good agreement with experiments (except near the bottom channel wall due to the use of an adiabatic boundary condition) and observed the sensitivity of thin laminar recirculation bubbles on the cube surface to near-wall resolution. Field measurements around Shinjuku district heating and cooling system in Tokyo, followed by computer simulations using STAR-CD (a commercial CFD code from CD-Adapco) with the standard $k-\varepsilon$ turbulence model including buoyancy effects by Huang et al. (2005), showed vertical thermal stratification around buildings and the ground. Though their simulations agreed with their experiments, it should be noted that very few field mea- 
surements were obtained, so it is difficult to assess the robustness of the simulations. The most recent 3D study was by Yang and Shao (2008) on a street canyon of varying aspect ratio $(0.25 \leq h / w \leq 2)$, in which the roughness elements were arranged in an aligned fashion. They computed the flow and dispersion characteristics of concentration from an area source released from the bottom surface. Whilst they discussed the behaviour of the flow and scalar flux profiles at different locations, the crucial question regarding resolution of the very thin thermal concentration layer at the surface was not addressed. It is to be noted that all the above mentioned literature primarily aimed at quantifying the heat released from the outer surfaces of the buildings. There is a considerable literature on internal ventilation and heat transfer (see Ji et al., 2009 for a recent example) and there is no doubt that in some situations the internal processes can affect, for example, the external heat transfer. This is less likely with fully insulated building surfaces and is not considered further in the present work.

There has as yet been no clear demonstration that current computational models can cope adequately at realistic Reynolds numbers with cases in which wall-to-fluid transfer processes are important. It is likely, for example, that given the thin thermal (or, analogously, pollutant) boundary layers at the building surfaces, noted in the field measurements mentioned earlier, the use of relatively coarse grids along with standard log-law formulations will be inadequate. The eventual objective of our current work is to be able to simulate heat fluxes from urban-like roughness elements by resolving accurately (or adequately parameterising) the thin thermal layers on the element surfaces. One of the means to achieve this is by using appropriate wall models for the flow and/or the scalar. The scalar wall models might again differ from case to case. For instance, the dynamics in the neutrally buoyant case are different from those in stable and unstable cases. Therefore, as a first step in meeting the objective of parameterising the scalar flux, a passive scalar dispersion test case has been studied and is presented in this paper. This case is the wind-tunnel experiment undertaken by Pascheke et al. (2008) who measured, both within and above the roughness canopy, scalar concentrations arising from a bottom-surface area source provided by a naphthalene sublimation technique. Unlike cases of elevated sources, surface source dispersion occurs first across the very thin wall layers and is thus analogous to heat transfer problems, at least in cases when temperature differences are not large enough to influence the flow dynamics. Simulation models are unlikely to be able to predict large temperature difference, buoyancy-affected cases adequately unless they are successful in the simpler cases of scalar transfer from walls.

We have performed 3D LES of this passive scalar dispersion within the two different urban-like geometries used by Pascheke et al. (2008) and the computational results are presented here and compared with the experimental data where possible. LES was used as this has proved to be a promising tool by many researchers and, in fact, is arguably the simplest approach for adequate computation of such unsteady, highly turbulent, complex geometry flows. Kanda et al. (2004), for example, used LES on simple cube arrays and obtained good agreement with the experiments and a detailed investigation of turbulent organized structures in aligned and staggered cube arrays using LES was subsequently reported by Kanda (2006). Xie and Castro (2006) compared LES and RANS for the flow past wall-mounted obstacles and observed that RANS performance is poor, especially within the canopy region. In 
contrast, they found satisfactory results using LES as compared to direct numerical simulations (DNS) by Coceal et al. (2006) at a Reynolds number of $R e=5000$ (based on building height and maximum mean velocity). Furthermore, a more recent LES (Xie and Castro, 2009) of a field site in central London showed very good agreement with experimental data for scalar concentrations from an elevated source (for which the influence of surface boundary conditions and Reynolds number are much less important).

The scope of the paper is as follows. Section 2 explains the numerical details and specifics of the computational domain, grid convergence issues are explored in Section 3 and Section 4 presents the mean concentration fields, comparing with experiments where possible. Section 5 discusses some of the important quantities (like turbulent scalar flux data) not generally available from laboratory experiments or RANS simulations, as this illustrates one of the significant advantages of using LES methodology. Concluding remarks are given in Section 6.

\section{Numerical Details and Settings}

\subsection{Flow equations and boundary conditions}

The filtered continuity and Navier-Stokes equations governing unsteady incompressible flow are

$$
\begin{gathered}
\frac{\partial u_{i}}{\partial x_{i}}=0 \\
\text { and } \frac{\partial u_{i}}{\partial t}+\frac{\partial u_{i} u_{j}}{\partial x_{j}}=-\frac{1}{\rho}\left(\frac{\partial p}{\partial x_{i}}+\delta_{i 1} \frac{\partial\langle P\rangle}{\partial x_{1}}\right)+\frac{\partial}{\partial x_{j}}\left(\frac{\tau_{i j}}{\rho}+v \frac{\partial u_{i}}{\partial x_{j}}\right)
\end{gathered}
$$

The resolved-scale velocity and pressure are respectively given by $u_{i}$ and $p$ with $u, v$ and $w$ the streamwise, lateral and vertical velocity components respectively. The flow was driven by a constant streamwise pressure gradient $\partial\langle P\rangle / \partial x, \delta_{i 1}$ is the Kroneckerdelta, and $\rho$ and $v$ are the density and kinematic viscosity of the fluid. $\tau_{i j}$ is the subgrid-scale (SGS) Reynolds stress and was handled using the Smagorinsky model in conjunction with a Lilly damping function near the walls. Smagorinsky's constant $C_{S}$ was chosen as 0.1 .

In the streamwise $(x)$ and lateral $(y)$ directions, periodic boundary conditions were employed. Stress free conditions were imposed on the top of the domain, i.e.,

$$
\frac{\partial u}{\partial z}=\frac{\partial v}{\partial z}=0 ; \quad w=0
$$

No slip conditions were set on the bottom surface $(z=0)$ and on all faces of the roughness elements. 
2.2 Scalar equation and boundary conditions

The filtered governing equation of the scalar is

$$
\frac{\partial c}{\partial t}+\frac{\partial u_{j} c}{\partial x_{j}}=\frac{\partial}{\partial x_{j}}\left(\left(k_{s}+k_{m}\right) \frac{\partial c}{\partial x_{j}}\right)
$$

where $c$ is the resolved-scale concentration of the scalar. $k_{s}$ is the subgrid turbulent diffusivity and is given by $v_{s} / S c_{s}$ where $v_{s}$ is the subgrid viscosity and $S c_{s}$ is the subgrid Schmidt number whose value was set to 0.9 (typical of the value used for the large scale field in RANS methods and in earlier LES studies - e.g. Xie et al. (2004); Cai et al. (2008)). $k_{m}$ is the molecular diffusivity and is defined as $v / S c_{m}$. The molecular Schmidt number $\left(S c_{m}\right)$ was taken as 2.284 , which is the value for naphthalene used as the scalar in the experiments. The naphthalene was coated onto the $z=0$ surface of one of the repeating units of size $0.08 \times 0.08 \mathrm{~m}^{2}$ (containing sixteen roughness elements). Therefore, in our current study, the same arrangement was used and is illustrated in Fig. 1 (shaded region), which shows views of the bottom of the computational domain. A constant concentration of the scalar was specified on the surface over the region $z / h=0,-4 h \leq x, y \leq 4 h$, where $h$ is the mean height of the roughness elements. The origin coordinates and the size of the area source region are chosen to be consistent with the experiments. Cyclic boundary conditions were employed in the streamwise and in the lateral directions and near the inlet, a plane with zero concentration was specified. This latter arrangement overrides the cyclic boundary condition at the inlet, but avoids having to specify an outlet boundary condition (like, for example, a zero gradient condition). On the upper surface, the normal gradient of the scalar was set to zero.

A finite volume approach was followed to discretise the flow and scalar equations. While a second-order central difference scheme was used for spatial discretisation of (1), the MARS (monotone advection and reconstruction scheme) scheme (STAR-CD, 2008) with a blending factor of 0.99 was used for (2) to avoid negative concentration values for the scalar. A second-order backward implicit scheme was used for discretising the time-dependent term.

The computational domain consisted of hexahedral cells and was of size $L_{x} \times L_{y} \times$ $L_{z}=16 h \times 16 h \times 6 h$ for C10S (uniform height cubes arranged in staggered fashion) and $16 h \times 16 h \times 10 h$ for RM10S (random height cuboids arranged in staggered fashion), where $h=0.01 \mathrm{~m}$ and $(x / h, y / h=0,0)$ is the centre of the source area. In each repeating unit, RM10S has five different heights that are equally spaced from 0.0028 $\mathrm{m}$ to $0.0172 \mathrm{~m}$ and approximately follow a Gaussian distribution with a mean height of $0.0102 \mathrm{~m}$ and standard deviation of $0.0036 \mathrm{~m}$. Both these domains contained 64 roughness elements, as seen in Fig. 1. In their DNS study on flow past cubes of various domain sizes $(8 h \times 8 h \times 4 h, 4 h \times 4 h \times 6 h)$, Coceal et al. (2006) found negligible differences in the mean flow except (as anticipated) near the top of the domain. As this would have insignificant influence on passive scalar dispersion in the canopy region, a domain height of $6 h$ was chosen in our current study for $\mathrm{C} 10 \mathrm{~S}$. For the RM10S configuration, the domain height of $10 h$ was chosen as the tallest roughness element is of height $1.72 h$. With this domain height, Xie et al. (2008) obtained good agreement in the mean flow and turbulent statistics with the experiments of Cheng 


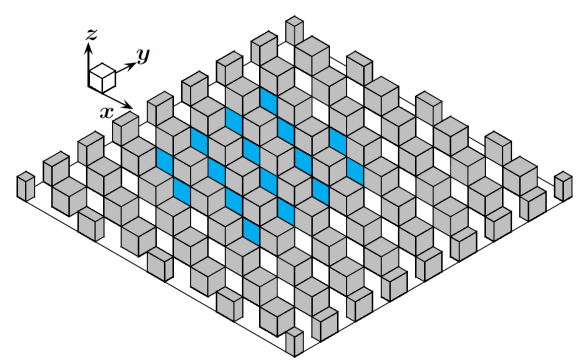

(a)

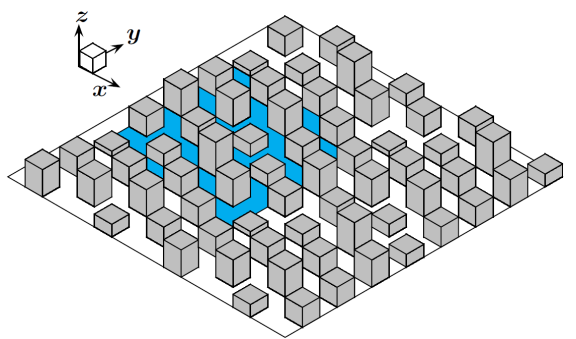

(b)

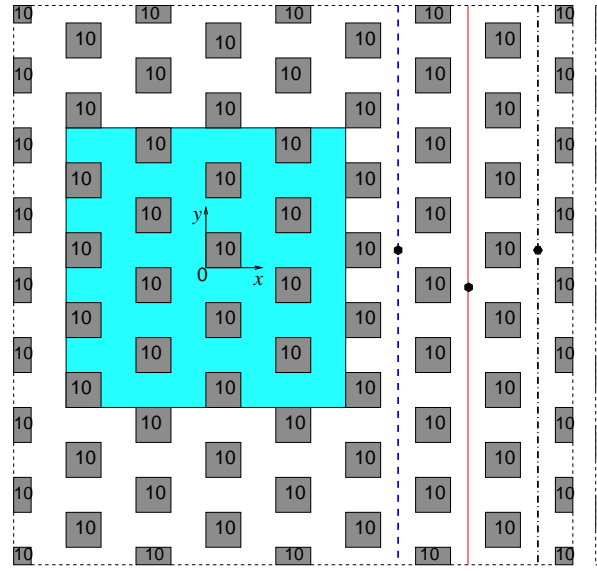

(c)

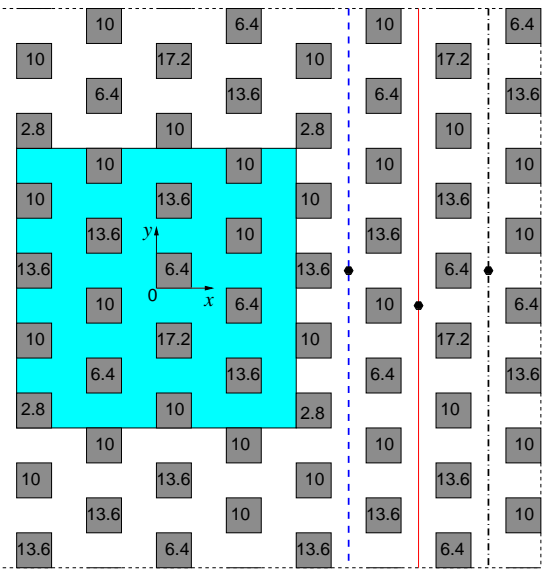

(d)

Fig. 1 Sketch of 3-D and plan view of C10S (a \& c) and RM10S (b \& d). The heights (in mm) of the blocks are indicated in the plan views. The scalar area source is represented by the shaded surface.

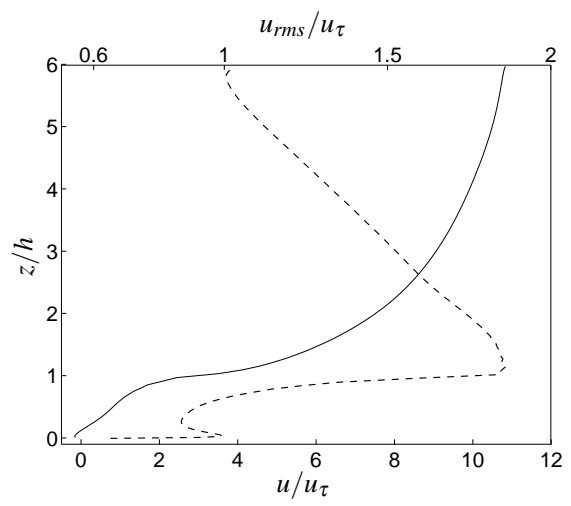

(a) $\mathrm{C} 10 \mathrm{~S}$

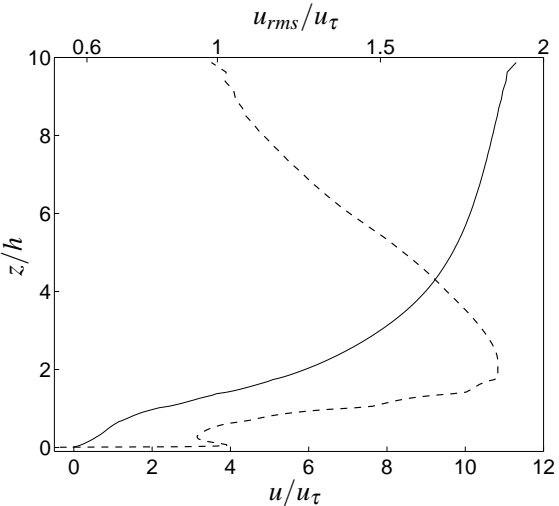

(b) RM10S

Fig. 2 Profiles of the streamwise mean velocity (solid line) and the streamwise r.m.s. velocity (dashed line) for C10S and RM10S. 
Table 1 Resolutions used in grid checks performed on a domain size of $4 h \times 4 h \times 6 h$

\begin{tabular}{|c|c|c|c|c|c|c|}
\hline \multirow{2}{*}{ Grid type } & \multirow{2}{*}{$\Delta x / h$} & \multirow{2}{*}{$\Delta y / h$} & \multicolumn{3}{|c|}{$\Delta z / h$} & \multirow{2}{*}{$z_{1}^{+}$} \\
\hline & & & $z / h \approx 0$ & $z / h=1$ & $z / h=6$ & \\
\hline Uniform grid (UG) & $1 / 16$ & $1 / 16$ & $1 / 16$ & $1 / 16$ & $1 / 16$ & 7.7 \\
\hline Non-uniform grid (NUG1) & $1 / 32$ & $1 / 32$ & $1 / 64$ & $1 / 32$ & $1 / 32$ & 2.1 \\
\hline Non-uniform grid (NUG2) & $1 / 32$ & $1 / 32$ & $1 / 128$ & $1 / 32$ & $1 / 16$ & 1.1 \\
\hline
\end{tabular}

and Castro (2002). The difference of domain heights in C10S and RM10S will have no significant effect on the scalar dispersion comparisons. This is because the mean flow from C10S agrees well with the DNS data from Coceal et al. (2007) on a domain size of $16 h \times 12 h \times 8 h$ and the scalar does not reach $z=6 h$, which together indicate that a domain height of $6 h$ is sufficient. The Reynolds number of the flow based on the mean obstacle height and the spatially averaged streamwise mean velocity at that height was around 2300 for C10S and 1900 for RM10S. Similar values were used in the experiments (Pascheke et al., 2008). The constant streamwise pressure gradient imposed on every cell was

$$
\frac{\partial\langle P\rangle}{\partial x_{1}}=\frac{\rho u_{\tau}^{2}}{L_{z}}
$$

where $u_{\tau}$ is the total wall friction velocity and the value of $\rho$ was chosen as 1.205 $\mathrm{kgm}^{-3}$. The initial duration of the simulations was $80 T$ (with $T=h / u_{\tau}$ ) for C10S and $120 T$ for RM10S and subsequent averaging times were $200 T$ and $400 T$ respectively. The time-step was small enough to require typically at least 25,000 steps for a $80 T$ averaging time and to ensure that the solutions were well-resolved in time. The spatial average of the streamwise velocity and the r.m.s. velocity for C10S and RM10S are shown in Figs. 2a and 2b. The maximum dispersive stress above the mean canopy height in the streamwise direction (not shown here) was observed to be around 0.3, when normalised by $u_{\tau}^{2}$. This could only be reduced significantly by using much longer averaging times, even assuming that the longitudinal rolls that produce such stresses move around laterally in time. However, we do not believe that this slight non-convergence in the outer flow will have a significant effect on the scalar transport in the canopy region. All the computations were carried out using STAR-CD (2008) version 4.08 on Iridis, a local supercomputer at the University of Southampton and on HECToR (High End Computing Terascale Resources), one of the machines at the UK's supercomputer centre.

\section{Grid checks}

Comparing with the DNS computations (Coceal et al., 2006) at $R e=5000$, Xie and Castro (2006) showed that a mesh containing 16 cells over each cube dimension was adequate to compute the flow past a staggered cube array (containing four cubes) using LES. It was argued that resolution of the thin boundary layers on the roughness elements was not necessary for good simulation of the flow, since form (i.e. pressure) drag dominated any viscous drag components in flows of this kind. We did not, however, expect such grids to be adequate for the concentration field arising from 
Table 2 Comparison of area averaged concentration values $\left(C_{N}^{*}\right)$ for each row of the roughness elements with the experiments.

\begin{tabular}{|c|c|c|c|c|c|}
\hline \multirow{2}{*}{$z / h$} & \multirow{2}{*}{$x / h$} & \multicolumn{4}{|c|}{$C_{N}^{*}$} \\
\hline & & UG & NUG1 & NUG2 & Expts \\
\hline \multirow{2}{*}{0.3} & -2 & 0.0328 & 0.0480 & 0.0460 & 0.0521 \\
\hline & 0 & 0.0500 & 0.0764 & 0.0762 & 0.0804 \\
\hline \multirow{2}{*}{1.2} & -2 & 0.0012 & 0.0029 & 0.0035 & 0.0026 \\
\hline & 0 & 0.0063 & 0.0119 & 0.0113 & 0.0105 \\
\hline
\end{tabular}

surface sources. Nonetheless, the scalar transport computations were started with this grid size (hereafter termed UG for uniform grid). In order to assess the grid dependence, finer grids were then used (on a smaller domain of size $4 h \times 4 h \times 6 h$, shown in Fig. 3); details are given in Table 1. The approximate values of the local near-wall cell location $\left(z_{1}^{+}\right)$ for all the grid types are also given in the last column of Table 1. It can be observed that the near wall cells are well within the viscous sublayer for

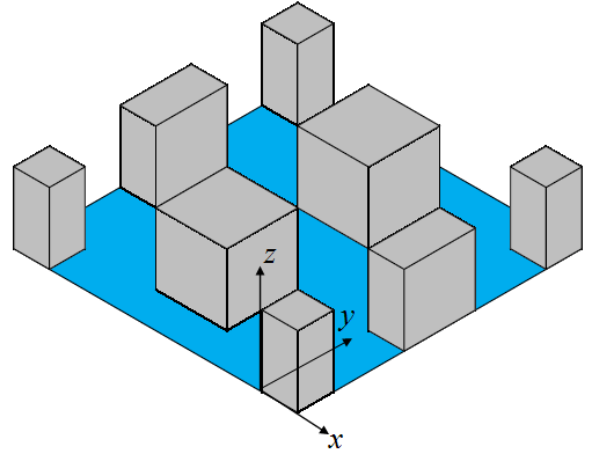

Fig. 3 Sketch of 3-D view of small computation domain used in grid checks.

non-uniform grids, NUG1 and NUG2, but not for the coarser uniform grid, UG. Unlike the large domain, this small domain has an area source all over the surface (at $z=$ 0 ). The boundary conditions for the flow and scalar are the same as those explained in Sect. 2. The area-averaged concentrations of the scalar normalised by the source concentration $\left(C_{N}^{*}\right)$ at different heights are shown in the Fig. 4. It is clear that, as expected, the coarser grid type UG is inadequate for computing the thin scalar boundary layer, whereas NUG1 and NUG2 both seem to perform well (at this Reynolds number); the latter grid size near the wall surface is almost equivalent to a DNS computation but this would of course be computationally prohibitive over a much larger domain.

Table 2 shows the area average concentration values of the scalar for each row of the roughness elements at two different heights for UG, NUG1, NUG2. (The details of how these values were obtained are explained in Sect. 4.1.) Although it is not really sensible to compare fine details of the simulated scalar field with the experiments primarily because the domain size is smaller and the passive scalar is released from all over the surface (which implies that, unlike in the experiments, lateral spreading is absent), Table 2 includes experimental values in order to demonstrate the influence of inadequate surface resolution. It can be deduced from the Table that the concentration values from UG are underestimated by $37 \%-54 \%$, whereas the values from NUG1 and NUG2 differ from experiments by $5 \%-13 \%$ (except for NUG2 at $x / h=-2, z / h=$ 1.2). The discrepancy of the finer grid simulations with the experiments could partly be due to the absence of lateral and streamwise scalar dispersion in the former. These grid checks confirm the importance of resolving the thin scalar layer near the surface. 


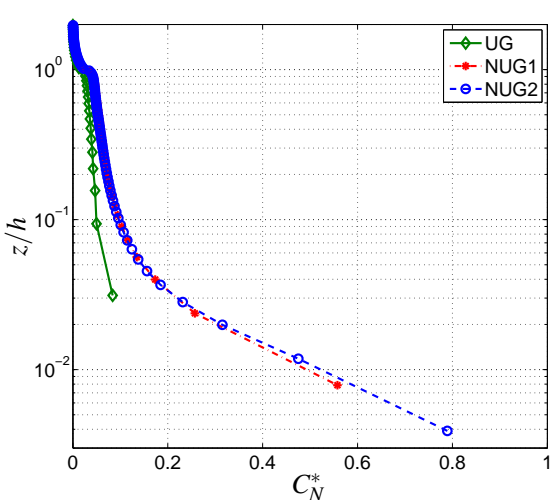

Fig. 4 Dependence of area averaged concentration values $\left(C_{N}^{*}\right)$ with the grid size.

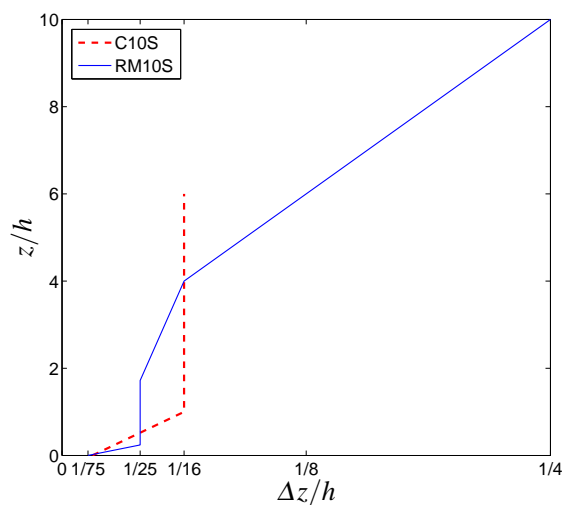

Fig. 5 Grid resolution in the $z$-direction. Near wall grid has $\Delta z / h=1 / 64$ for $\mathrm{C} 10 \mathrm{~S}$ and $1 / 75$ for RM10S. The variations immediately above are initially linear.

For both roughness cases the resolutions that were finally used in the $z$-direction are shown pictorially in Fig. 5. Between the two successive heights of different resolutions, the grid spacing followed geometric progressions. In the streamwise and lateral directions, $h / 16$ resolution was used throughout the domain. This gave overall cell counts of $6.7 \times 10^{6}$ and $8.4 \times 10^{6}$ for C10S and RM10S respectively. Various qualitative and quantitative comparisons with the experimental data and also the differences in the scalar transport processes in these two geometries are presented and discussed in the following sections.

\section{Mean flow data: comparisons with experiment}

\subsection{Concentration fields above the source area}

Contours of the mean concentration fields (normalised with the source concentration) for C10S and RM10S at $z / h=0.3$ and 1.2 are shown in Figs. 6 and 7 respectively. For $\mathrm{C} 10 \mathrm{~S}$, above the source area at $z / h=0.3$ (Fig. 6a), a regular pattern of regions of higher concentrations behind the cubes and lower concentrations in front of the cubes is observed. The former may be due to entrainment of large amounts of scalar into the cube wakes but more importantly to the trapping of scalar emitted on the surface below, whilst the latter is caused by the removal of scalar by the upstream flow. Although similar behaviour is observed for RM10S (Fig. 6b), the pattern is not regular, thus indicating strongly the differences in the transport processes for the two different surfaces. For example, the recirculation region in front of the tallest roughness element of height $0.0172 \mathrm{~m}$ has significant influence on the wakes of the upstream roughness elements. The lateral dispersion is nevertheless found to be more or less the same in these two geometries. At $z / h=1.2$, which is immediately above the canopy for C10S (Fig. 7a), higher concentration fields are observed around $x / h$ $=5.5, y / h=-0.5$ and $x / h=5.5, y / h=1.5$. These are caused by streamwise and 


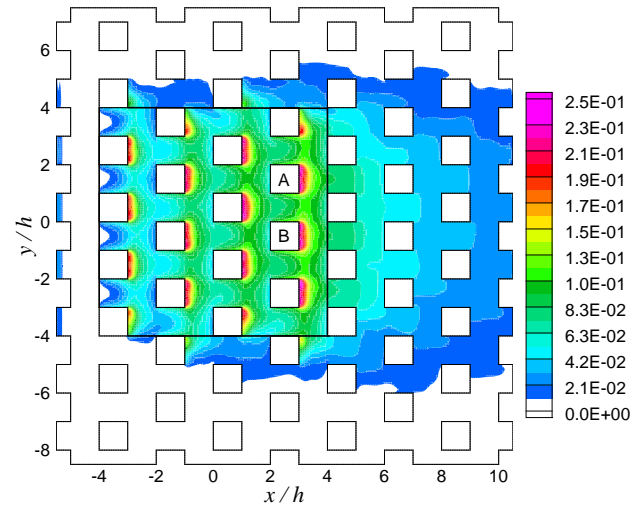

(a) $\mathrm{C} 10 \mathrm{~S}$

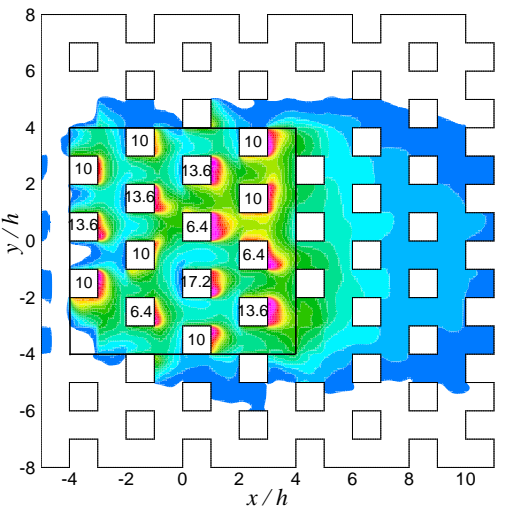

(b) RM10S

Fig. 6 Contours of $C_{N}^{*}$ at $z / h=0.3$ (square box represents the location of scalar on the surface).

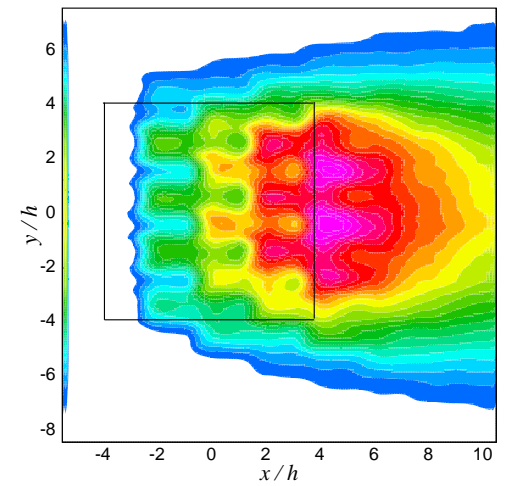

(a) $\mathrm{C} 10 \mathrm{~S}$
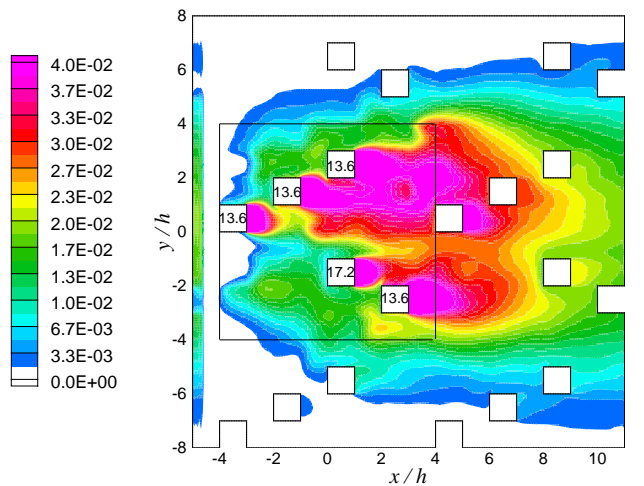

(b) RM10S

Fig. 7 Contours of $C_{N}^{*}$ at $z / h=1.2$.

vertical dispersion of the larger concentrations that are present within the canopy on the leeward side of cubes A and B identified in Fig. 6a. RM10S shows higher concentrations at this height, especially in the wakes of the tall roughness elements that are present within the area source region (Fig. 7b). This is a result of stronger vertical dispersion in the random height geometry compared to the uniform height geometry. Similar contours of concentration fields are observed in the experiments (Figs. 10 and 11 in Pascheke et al., 2008).

Quantitative checks with experiments are made by comparing the area-averaged concentrations obtained from each row of roughness elements above the source area; these are shown in Fig. 8b for two different heights. The symbol at $x / h=-2$ in Fig. $8 \mathrm{~b}$, for example, is obtained from the average of the concentration values from the region "A1" shown in Fig. 8a. The simulations show good agreement with the 


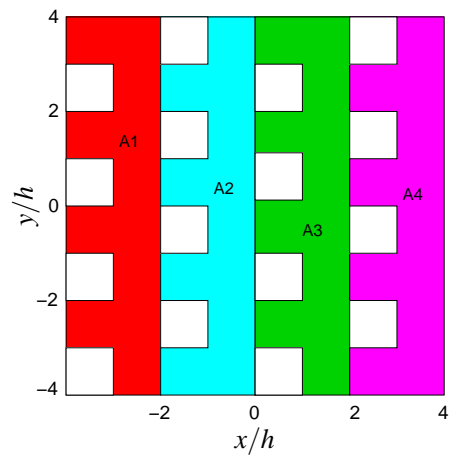

(a)

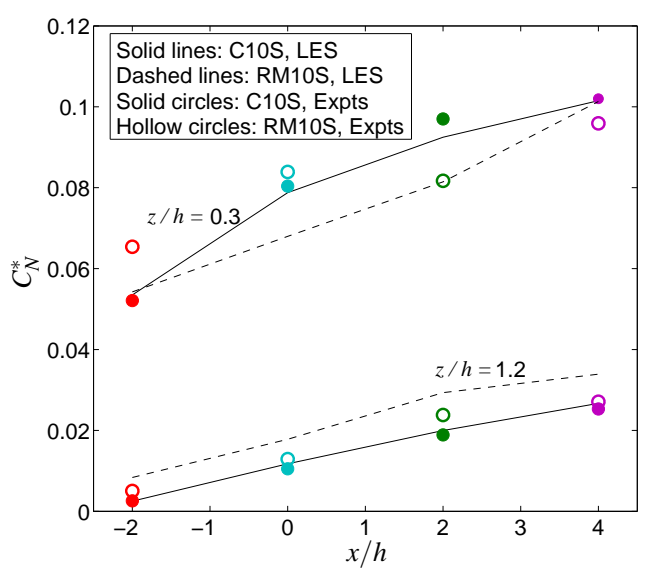

(b)

Fig. 8 (a) Area-averaged regions and (b) the corresponding concentrations above the source area.

experiments, especially for C10S. The higher $C_{N}^{*}$ values of RM10S compared to C10S at $z / h=1.2$ shows that the rapid vertical dispersion is stronger above the mean canopy height in the former case compared to the latter. It was observed in the experiments that at $z / h=0.3$, the difference in the area-averaged concentration for C10S and RM10S (for the whole unit) was less than $5 \%$ and at $z / h=1.2$, the area-averaged concentration was found to be $17 \%$ larger for RM10S. But the LES data suggest that the differences are about $6.5 \%$ at $z / h=0.3$ and about $32 \%$ at $z / h=1.2$. These discrepancies between the experiments and the simulations can partially be attributed to inaccurate location of the measurement probe (F. Pascheke, pers. comm, 2008) in the experiments - even small positional changes within the relatively steep gradients which occur at $z / h \approx 1.2$ could lead to significant differences. Other reasons could be due to $\pm 3 \%$ variation in the reproducibility of the concentration measurements from the average value and much more refined spatial averaging in the simulations than was possible in the experiments, which had only 75 measurement locations per region (i.e. in region A1, Fig. 8a). Note the non-monotonic variation of $C_{N}^{*}$ at $z / h=$ 0.3 in the experiments with RM10S; this would seem intuitively rather unphysical and may be a further indication of experimental uncertainties.

\subsection{Concentration profiles from the downstream of the source area}

More detailed quantitative checks are made by comparing the concentration profiles at various locations downstream of the source area $($ at $x / h=5.5,7.5,9.5)$ in the lateral and the vertical directions. These are shown in Figs. 9 and 10 respectively. Good agreement in lateral concentration profiles at $z / h=0.6$ is observed between LES and experiments for both C10S and RM10S. Due to the larger vertical dispersion in the RM10S case compared to C10S, relatively smaller amounts of the scalar are advected downwind of the area source at $z / h=0.6$ and hence the peak values in Fig. $9 \mathrm{~b}$ are 


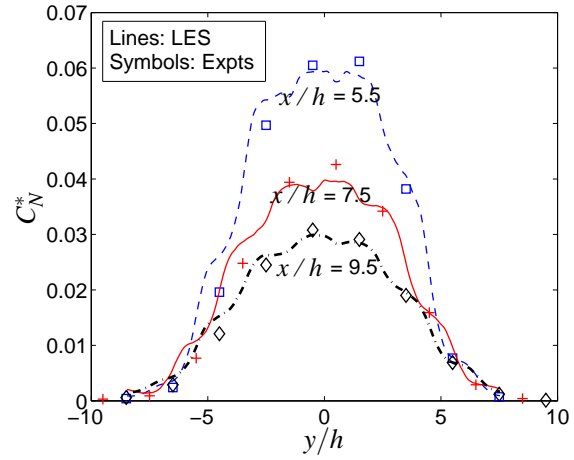

(a) $\mathrm{C} 10 \mathrm{~S}$

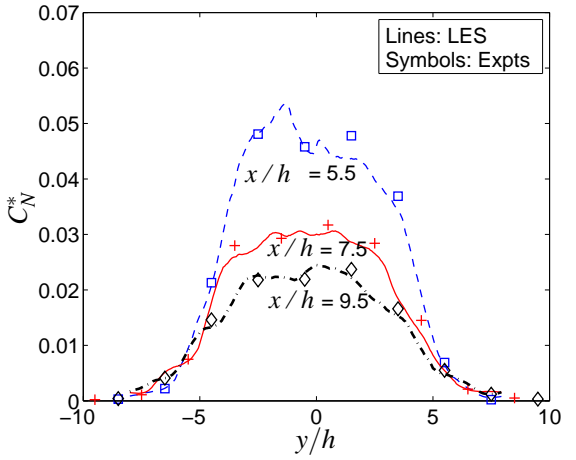

(b) RM10S

Fig. 9 Lateral concentration profiles from the downstream of the source area at $z / h=0.6$. The locations of these profiles are denoted by lines in Figs. 1c \& 1d.

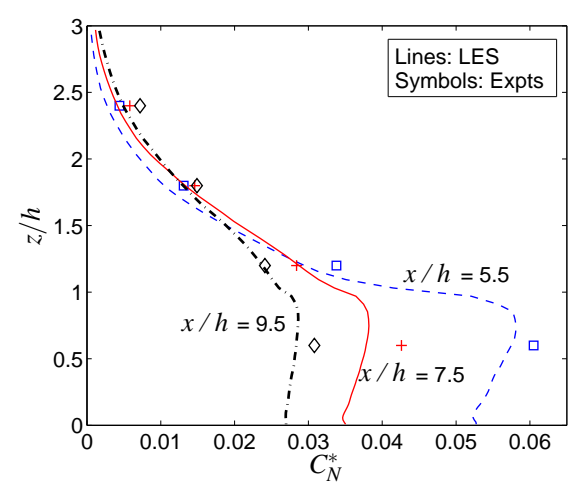

(a) $\mathrm{C} 10 \mathrm{~S}$

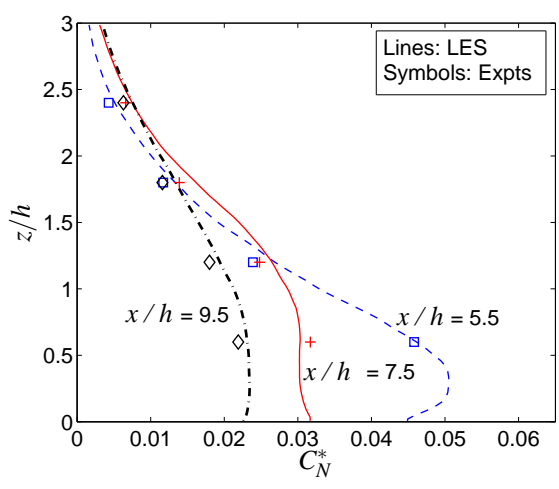

(b) RM10S

Fig. 10 Vertical concentration profiles downstream of the source area at $y / h=0.5($ at $x / h=5.5,9.5)$ or -0.5 (at $x / h=7.5$ ). The locations of these profiles are denoted by dots in Figs. 1c \& $1 \mathrm{~d}$.

smaller than those in Fig. 9a. All these profiles exhibit roughly Gaussian behaviour, especially at farther downstream distances from the source area.

In Fig. 10, quite good agreement in the vertical concentration profiles can again be observed between LES and experiments. For RM10S (Fig. 10b), the concentration values are lower in the vicinity of the bottom surface for the same reason as explained above. Also, the concentration gradients above the canopy are less steep compared to those in the C10S case (Fig. 10a) and this could be due to the strong streamwise advection. Similar observations were made by Kanda and Moriizumi (2009) in comparing data for a uniform block array with one which had a mixture of two different block heights. They speculated that the decrease in the advective velocity and the vertical temperature gradient (the scalar in their experiments was temperature) was due to the greater degree of aerodynamic mixing in the non-uniform-height array. 


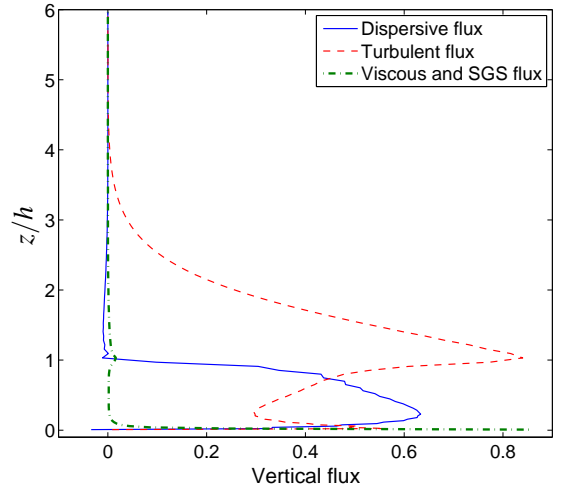

(a)

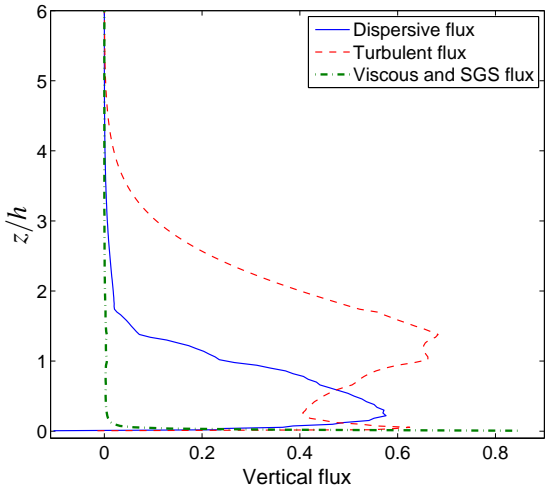

(b)

Fig. 11 Profiles of normalised vertical fluxes for (a) C10S and (b) RM10S.

\section{Scalar flux behaviour}

\subsection{Overall vertical fluxes}

A major advantage of computations like these (compared with RANS-type methods) is that the scalar flux from the mean flow, turbulent fluctuations and the molecular diffusion can all be determined explicitly; these are very difficult if not impossible to obtain from field or laboratory measurements. Figure 11 illustrates the variation of vertical flux quantities with height for both surfaces. The dispersive flux shown in this figure was obtained from $\langle\bar{c} \bar{w}\rangle-\langle\bar{c}\rangle\langle\bar{w}\rangle$, the turbulent flux from $\left\langle\overline{c^{\prime} w^{\prime}}\right\rangle$ and the viscous and subgrid-scale flux from $\left\langle\left(k_{s}+k_{m}\right)(\partial \bar{c} / \partial z)\right\rangle$, where \langle\rangle denotes spatial averaging, primes denote fluctuating quantities and the overline denotes time averaging. These quantities were calculated in the region $-4 \leq x / h \leq 10,-8.5 \leq y / h \leq 7.5$ (C10S), $-8 \leq y / h \leq 8$ (RM10S) and are normalised by the respective total surface flux from the area source. For both cases, the viscous and SGS flux is dominant only in the vicinity of the surface and becomes negligible within a very short height above the surface, as its key contribution is from molecular diffusion which falls rapidly with height. The vertical dispersive flux gradually increases with height from the surface and attains a maximum, coincidentally at $z / h \approx 0.23$ for both surfaces. This is then followed by a gradual decrease and it reaches zero near the maximum canopy height (0.01 $\mathrm{m}$ for C10S and $0.0172 \mathrm{~m}$ for RM10S) due to strong shear there. For C10S, the turbulent flux attains a local maximum value very close to the surface (around $z / h=0.02$ ), then decreases with height and reaches a local minimum at around the same height where the dispersive flux becomes maximum, before increasing again until it reaches its maximum value around the canopy height and then eventually decreases again with height. For RM10S, the behaviour is similar to the C10S case up to the mean canopy height. Above the mean height, due to the presence of other tall roughness elements, the behaviour becomes non-monotonic until about $z / h=$ 


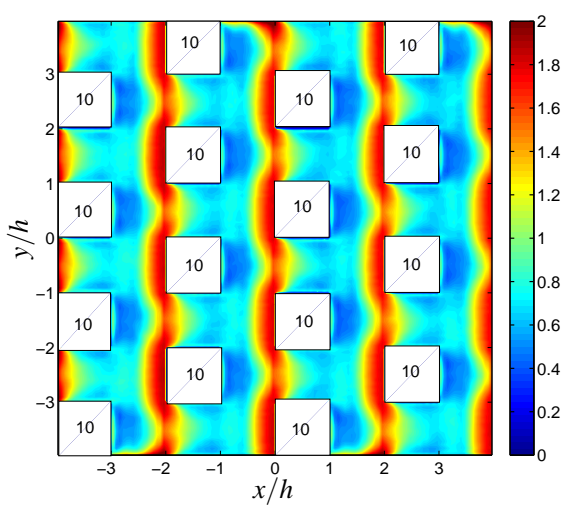

(a) $\mathrm{C} 10 \mathrm{~S}$

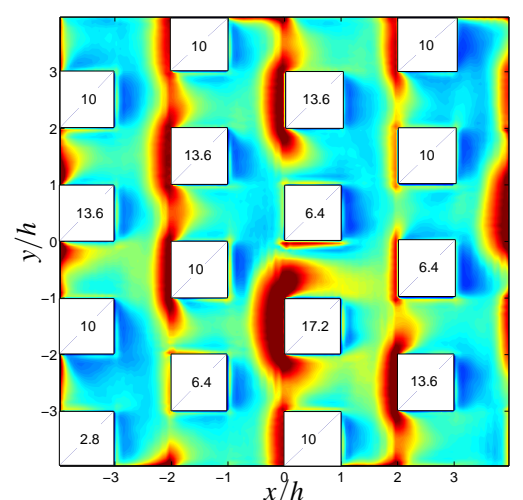

(b) RM10S

Fig. 12 Contours of the vertical viscous and SGS flux from the region $-4 \leq x / h, y / h \leq 4$ and at $z / h \approx$ 0.007 .

1.38 and then the flux gradually reduces to zero with increasing height, but at a lower rate compared to $\mathrm{C} 10 \mathrm{~S}$.

It is observed that for $\mathrm{C} 10 \mathrm{~S}$, the vertical flux across the plane $z / h \approx 1.5$ is some $50 \%$ of the total vertical flux from the source, with the remaining $50 \%$ being advected downstream; for RM10S this balance is observed at $z / h \approx 1.8$. The total flux from the surface obtained from LES is found to differ from the experimental values by about $3.6 \%$ for $\mathrm{C} 10 \mathrm{~S}$ and about $16 \%$ for RM10S. Both the experiments and the simulations nonetheless showed larger surface flux values for the geometry with uniform heights. Higher turbulence levels in the near wall region are evident in Fig. 2 and these imply better mixing and hence smaller concentration gradients compared to those in C10S. This could be the reason for the lower surface flux values in RM10S. Whilst the experiments showed the surface flux for C10S to be $3 \%$ higher than RM10S, the simulations estimated it to be $16.8 \%$ (the surface flux values are normalised with the free stream velocity, $\left.U_{r e f}\right)$. This difference between the simulations and experiments is not small and can perhaps be accounted for by a number of factors - measurement uncertainties, differences in upstream conditions, etc.

\subsection{Surface flux distribution near the source area}

In the experiments, a higher mass flux was noticed around the periphery of the source area (Barlow, pers. comm, 2009). To observe this in LES, contours of the normalised vertical viscous and SGS flux immediately above the source area (where it dominates turbulent fluxes, see Fig. 11) are plotted for C10S and RM10S in Fig. 12. The first and foremost inference that can be drawn is that this near-wall flux distribution is quite different for the two surfaces. In C10S, the flux values are about twice as large in a few specific regions around the lateral edges of the area source, i.e. at $y / h=$ -4 and 4 , when compared to the other $y / h$ locations. This can be seen quantitatively by comparing, for example, the region $-3 \leq x / h \leq-2, y / h=-4$ with the same $x / h$ 


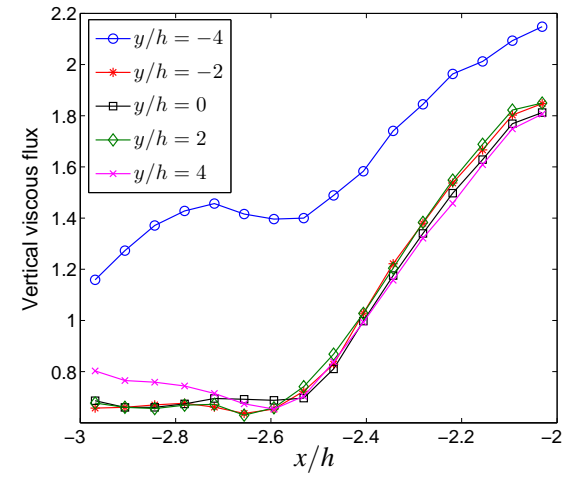

(a)

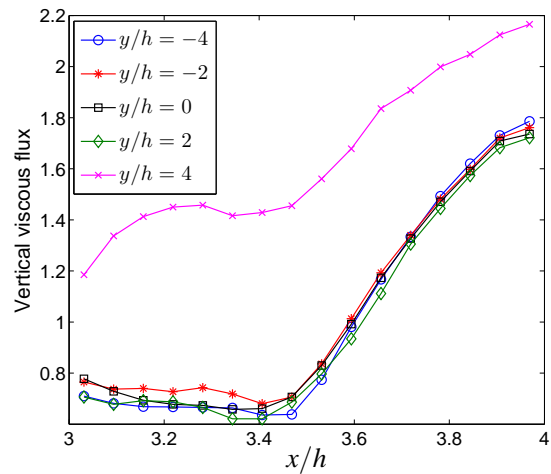

(b)

Fig. 13 Vertical viscous and SGS flux profiles of C10S from different locations at $z / h \approx 0.007$. The locations of these profiles are denoted by solid lines in Fig. 16a.

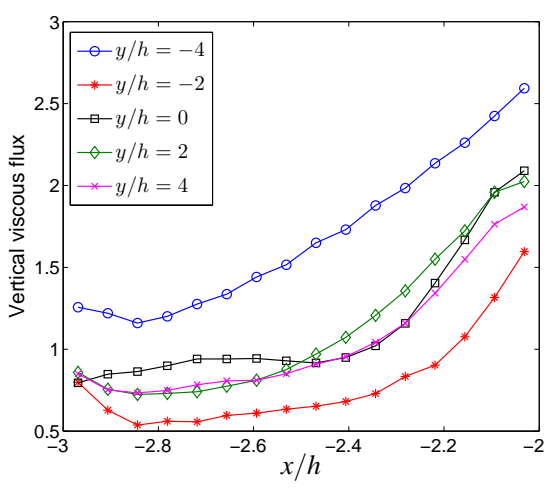

(a)

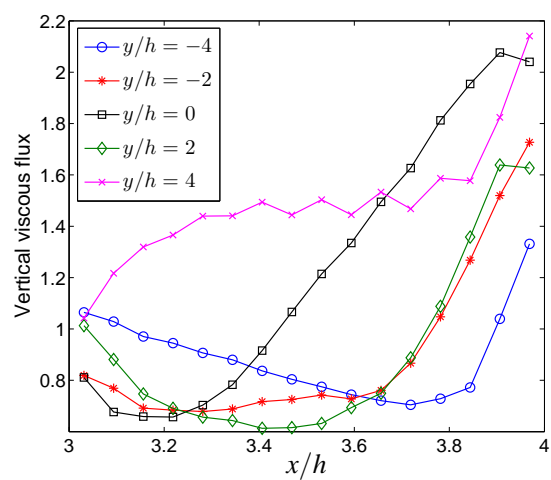

(b)

Fig. 14 Vertical viscous and SGS flux profiles of RM10S from different locations at $z / h \approx 0.007$. The locations of these profiles are denoted by solid lines in Fig. 16a.

region at $y / h=-2,0,2$ and 4 , as shown in Fig. 13a. The locations of these profiles are indicated by solid lines in Fig. 16a. Similar observations can be made by comparing $3 \leq x / h \leq 4$ at $y / h=4$ with the corresponding $x / h$ locations at $y / h=2,0,-2$ and -4 as shown in Fig. 13b. These observations therefore confirm the experimental findings. Similar behaviour is observed in the RM10S case (Fig. 14). The surface flux along $y / h=0$ dominates the surface flux along $y / h=4$ in the region $3.6<x / h<4$ due to the presence of the second tallest roughness element (i.e. the $0.0136 \mathrm{~m}$ block at $x / h$ $=4, y / h=0)$, as evident in Fig. 14b.

In C10S, at $x / h=-4$, i.e. at the leading edge of the area source and in front of the obstacles, higher flux values are observed. This is because the oncoming "cleaner" flow carries the scalar in front of the block into the small recirculation region illustrated by the mean velocity vectors $(u, w)$ in the $x-z$ plane in the region $1 \leq x / h \leq$ $2,0 \leq z / h \leq 1$ shown in Fig. 15. Also, in the wake of the obstacles in C10S, flux val- 


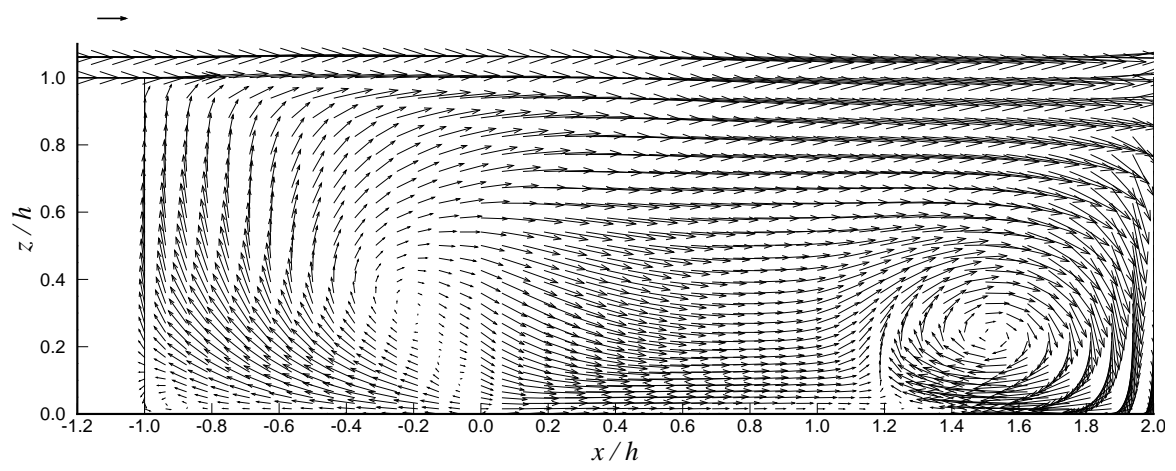

Fig. 15 Mean velocity vectors on the $x-z$ plane at $y / h=-2.5$ between cubes in C10S. All arrows have lengths scaled to that of the defining arrow at top left, for which $u / u_{\tau}=1.0$. The location of this plane is denoted by a dashed line in Fig. 16a.

ues are low as the scalar is entrapped in the larger recirculation region. But very close to the obstacle in the wake region, the flux values are relatively higher and this arises due to the secondary separation of the flow. This tiny secondary separation bubble in the region $-1 \leq x / h \leq-0.8,0<z / h<0.1$ can just be identified in Fig. 15. Unlike C10S, the flux pattern is irregular in RM10S because of the randomness in the heights of the obstacles. The flux values are found to be significantly larger in front of the tallest roughness elements $(0.0172 \mathrm{~m}$ and $0.0136 \mathrm{~m})$ in comparison with the remaining elements. At $x / h=-4$, flux is more prominent on either side of the 0.0136 m obstacle compared to the rest and a similar pattern is observed at $x / h=4$, no doubt also due to the randomness in obstacle height.

\subsection{Vertical flux distribution in typical regions}

To gain a better insight into the distribution of vertical flux in the two cases, three different zones of size $h \times h$ were identified within the area source unit. They are "behind", "in front" and in the "gap" for each of the obstacles, as shown in Fig. 16a for a single obstacle. The average of the vertical flux (i.e. advective flux, $\langle\bar{c} \bar{w}\rangle+$ turbulent flux, $\left\langle\overline{c^{\prime} w^{\prime}}\right\rangle$ ) profiles in each of these zones has been calculated for both the geometries and is shown in Fig. 16b. Each profile has been normalised by the respective total surface flux value. Note that all the profiles show values close to zero at the near-wall point - they are identically zero at the wall, but the computation does not explicitly include points on the wall. The profiles from "behind" dominate the others in both geometries due to the greater entrainment of the scalar in the large recirculation regions. The profiles from "in front" have lower flux values compared to the "gap" profiles within the canopy (but only up to $z / h \approx 0.64$ in RM10S) and this situation reverses just above the mean canopy height. The sum of the profiles from these three regions for RM10S is found to be large above the mean canopy height compared to C10S. This indicates that the taller roughness elements generate 


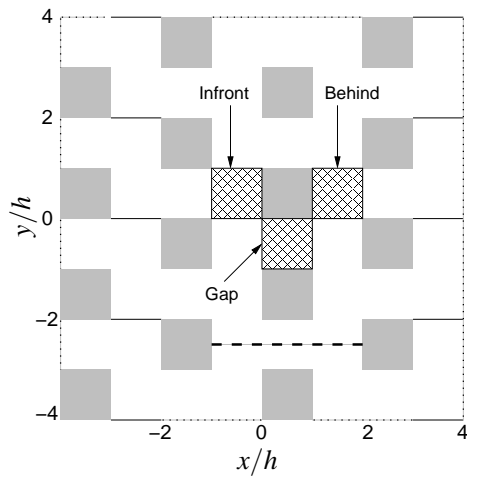

(a)

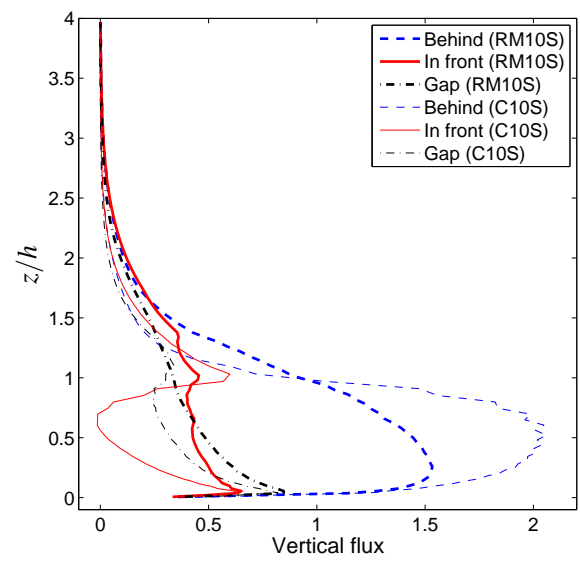

(b)

Fig. 16 (a) Solid lines represent the locations of the profiles shown in Figs. 13 and 14; dashed line represents the location of the vector plane shown in Fig. 15. Three typical zones are indicated by the crosshatched lines around a block and (b) Sum of the normalised advective and turbulent vertical flux profiles from these zones.

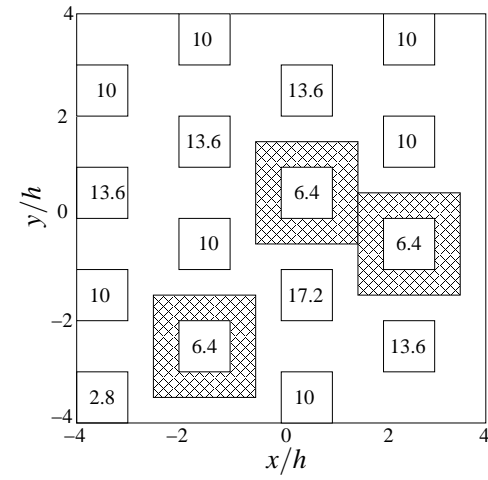

(a)

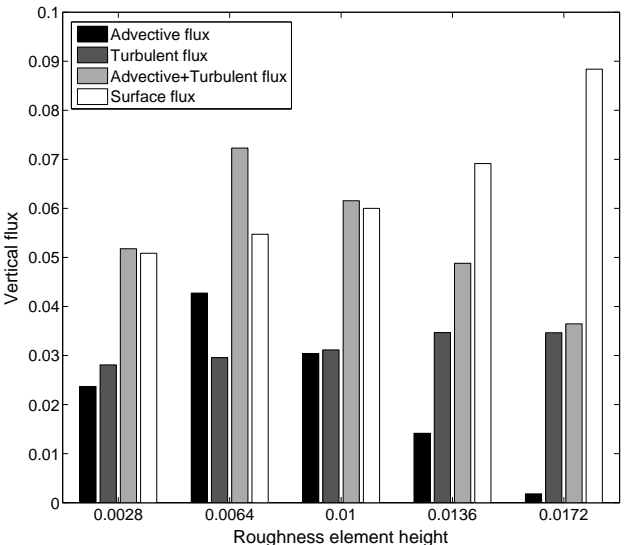

(b)

Fig. 17 (a) The regions used to calculate the flux around $0.0064 \mathrm{~m}$ block (note that the block heights are given in $\mathrm{mm}$ in the plan view) are shown by cross-hatched lines. Similar regions are used around other blocks to calculate the flux. (b) The vertical fluxes shown are the advective flux, turbulent flux, their sum at $z / h \approx 0.1$ and the surface flux at $z / h \approx 0$.

a relatively larger vertical flux above the mean canopy height which could be due to the weaker streamwise advection when compared to $\mathrm{C}_{10 \mathrm{~S}^{1}}$.

1 A referee suggested that the larger vertical flux above the RM10S canopy is more likely caused by less mutual sheltering between the blocks. This certainly seems possible. 
5.4 Flux contribution by each random height roughness element

It was shown by Xie et al. (2008) that $65 \%$ of the total surface drag in the RM10S case is contributed by the roughness elements of height $0.0136 \mathrm{~m}$ and $0.0172 \mathrm{~m}$, even though their frontal area is only $45 \%$ of the total. It is thus also interesting to examine the flux contribution near each of these roughness elements. Therefore, a region delineated by a width of $0.5 h$ around the sides of each block was chosen, giving a square region of size $2 h \times 2 h$; Fig. 17a shows the regions for the three 0.0064 $\mathrm{m}$ blocks. The horizontally averaged vertical turbulent and advective flux values were obtained over these three regions, normalised by the total surface flux. These were calculated at $z / h=0.097$, where the viscous and the streamwise flux contributions were found to be negligible. In a similar manner, the flux values around the remaining blocks were obtained and the results for all block (height) categories are shown in Fig. 17b. The advective flux is higher for the $0.0064 \mathrm{~m}$ blocks than for the $0.0028 \mathrm{~m}$ blocks and it is lower for the higher blocks. Unlike the advective flux, the turbulent flux is nearly constant for all the roughness elements. The turbulent flux is found to be significantly larger than the advective flux around the 0.0136 and $0.0172 \mathrm{~m}$ blocks. The sum of the advective and turbulent flux follows the same behaviour as that of the advective flux and is included in the figure; the contribution of the surface flux normalised by the total flux near each of the roughness elements is also included. This figure clearly shows the increase in the surface flux contribution with the increase in block height. An interesting feature is that the sum of the advective and turbulent fluxes around the $0.0064 \mathrm{~m}$ blocks exceeds the corresponding surface flux. This is due largely to the greater advective flux around the $0.0064 \mathrm{~m}$ block at $(x / h, y / h)=(0,0)$, which is higher by some $20 \%$ and $15 \%$ than those around the blocks at $(2,-1)$ and $(-2,-3)$, respectively. It can therefore be deduced that the tall roughness elements have significant influences on the flux distribution of the scalar.

\subsection{Estimation of eddy diffusivity}

From vertical turbulent flux data it is possible to estimate the eddy diffusivity, defined in the usual way as the ratio of the turbulent flux to the mean concentration gradient, $-\overline{c^{\prime} w^{\prime}} /(\partial \bar{c} / \partial z)$. The resulting spatially averaged values, normalised by $u_{\tau} h$, are shown in Fig. 18a. Data are shown only up to $z / h=3$ as concentrations are very small above this height. For C10S, the eddy diffusivity values are larger within the canopy than they are for RM10S. Due to the strong shear layer immediately above the mean canopy height, there is a sharp decrease in the concentration gradient and a slight decrease in the turbulent flux. As a result, the eddy diffusivity reaches a local minimum value of 0.08 . In the case of RM10S, the eddy diffusivity has four such local minimum values (ranging from 0.13 to 0.25 ), each occurring at the four largest roughness element heights; the $0.0028 \mathrm{~m}$ block, however, appears not to yield a significant peak. Above the mean canopy height, the eddy diffusivity is generally a little larger for RM10S, due to the relatively larger turbulent fluxes.

Figure $18 \mathrm{~b}$ compares the eddy diffusivity averaged over the more localised regions surrounding the individual blocks defined in Sect. 5.4, within the area source 


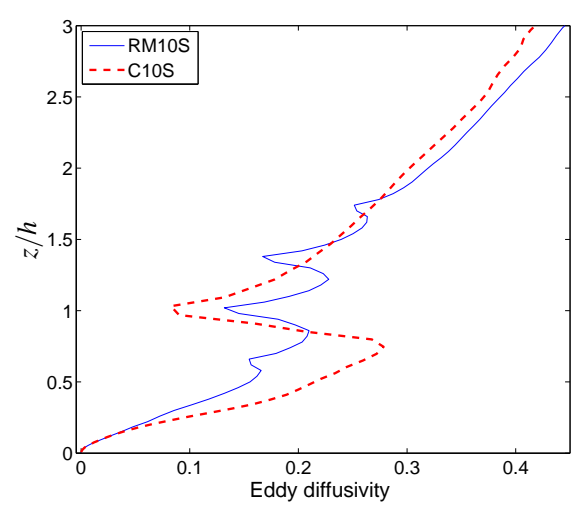

(a)

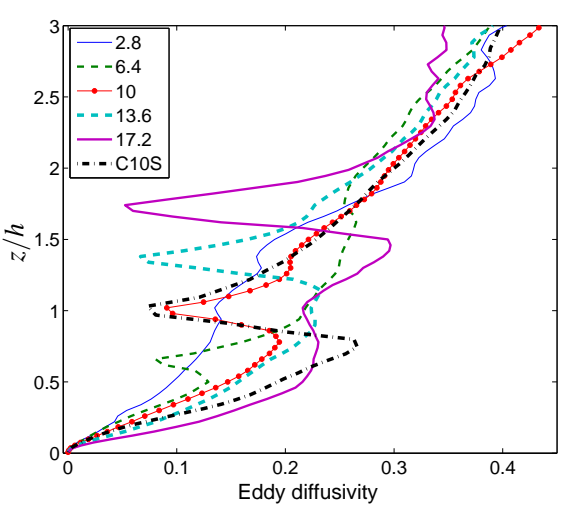

(b)

Fig. 18 (a) Comparison of eddy diffusivity in two geometries (b) eddy diffusivity averaged over local regions (e.g. Fig. 17a) for RM10S within the source area.

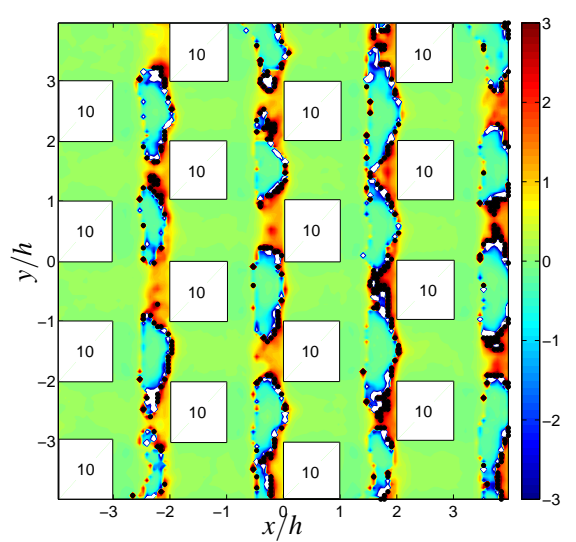

(a) $\mathrm{C} 10 \mathrm{~S}$

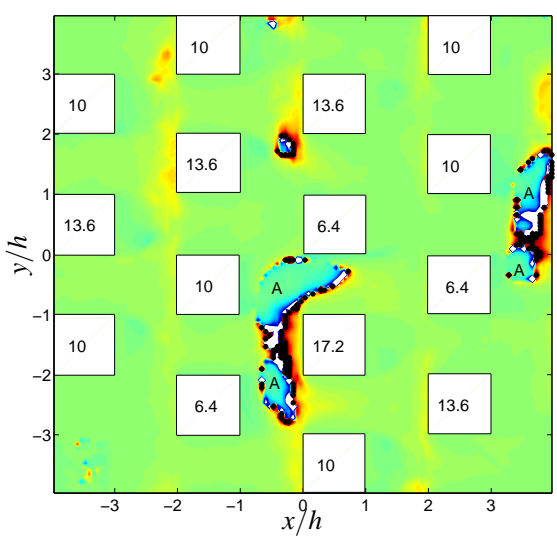

(b) RM10S

Fig. 19 Contours of eddy diffusivity from the region $-4 \leq x / h, y / h \leq 4$ and at $z / h=0.3$. This region is above the area source shown in the Fig. 1. The black and white areas indicate regions where the eddy diffusivity exceeded 3 or was below -3 , respectively

region $(-4 \leq x, y \leq 4)$. As mentioned above, local minimum values are observed at each height of the roughness element (except $0.0028 \mathrm{~m}$ block). Surprisingly, all the local minimum values are in the range 0.05 to 0.08 . This is due to large local concentration gradients at the height of each of the block coupled with weak turbulent fluxes. The concentration gradient is large because the recirculation regions behind the blocks retain large amounts of scalar which is eventually removed by the strong shear layer at the block height.

The eddy diffusivity is commonly modelled as the ratio of the turbulent viscosity $v_{t}$ to the Schmidt number of the scalar. Many RANS models, particularly those based on $k-\varepsilon$ type turbulence models, employ a turbulent viscosity model defined 


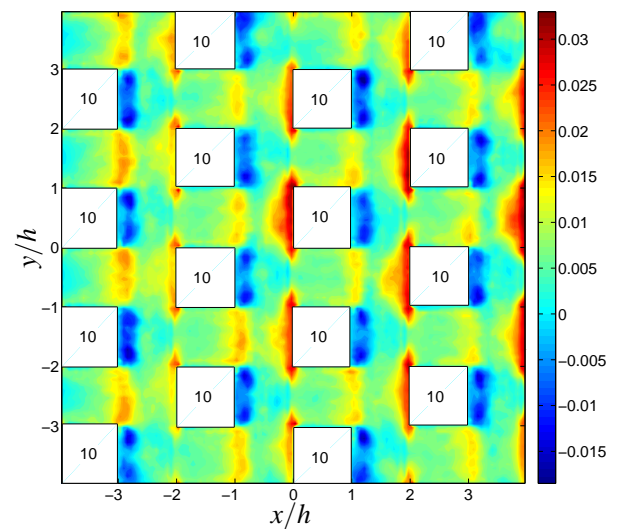

(a) $\mathrm{C} 10 \mathrm{~S}$

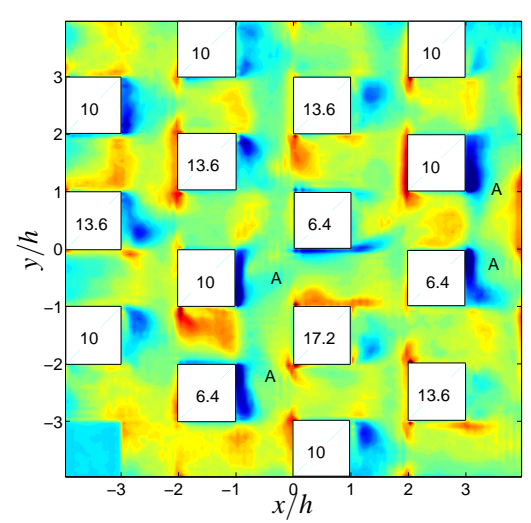

(b) RM10S

Fig. 20 Contours of turbulent flux $\overline{c^{\prime} w^{\prime}}$ in the region $-4 \leq x / h, y / h \leq 4$ at $z / h=0.3$.

by $v_{t}=-\overline{u^{\prime} w^{\prime}} /(\partial u / \partial z)$ with the viscosity often given by $v_{t}=C_{\mu}\left(k^{2} / \varepsilon\right)$, where $C_{\mu}$ is a constant and the kinetic energy $k$ and dissipation rate $\varepsilon$ are obtained by solving their modelled transport equations. The value of $v_{t}$ is thus inevitably positive and hence the resultant eddy diffusivity must always be positive. But the contours presented in Fig. 19 for the region $-4 \leq x / h, y / h \leq 4$ at $z / h=0.3$ show that although the spatially averaged values are positive (Fig. 18a) locally negative values occur quite often. Interestingly, these negative values do not necessarily arise from a negative turbulent flux (i.e. negative $\overline{c^{\prime} w^{\prime}}$ ) but from positive concentration gradient (i.e. scalar concentration locally increasing with height). To illustrate this, the turbulent flux contours are shown for the same region in Fig. 20. For example, the eddy diffusivity at $(x / h, y / h)=(-2.4,0.09)$ in C10S is -0.34 whereas the turbulent flux is $\approx$ 0.006 and in RM10S, at $(x / h, y / h)=(0.344,-0.344)$, the turbulent flux is $\approx 0.007$ whereas the eddy diffusivity is -0.43 . The most dominant regions of negative diffusivity for RM10S, labelled 'A' in Figs. 19b and 20b, occur around the tallest block, but there are similar regions just upstream of one of the next tallest blocks $(0.0136$ $\mathrm{m})$. Such regions of negative eddy diffusivity gradually disappear with an increase in height. However, the fact that they exist and can be quite extensive suggests that classical RANS modelling for the scalar would fail to capture significant features of the complex pollutant field. Such regions of counter-gradient diffusion have also been observed within vegetation canopies (Finnigan, 2000).

\section{Conclusions}

The dispersion of a passive scalar area surface source from two different urban-like geometries that have the same plan area and frontal area densities $\left(\lambda_{p}=\lambda_{f}=25 \%\right)$ has been investigated using large eddy simulations and compared with the laboratory experiments of Pascheke et al. (2008). Systematic grid checks have shown that for a surface area source the resolution has to be sufficiently fine to resolve the thin 
scalar boundary layer across which the scalar is transported. Simulations were done using a resolution of $h / 64$ near the area source for the C10S (the uniform height array) and $h / 75$ for the RM10S (an array of random height elements). Qualitative and quantitative comparisons between simulations and experiments showed reasonable agreement. Within the mean canopy height of the area source unit, large amounts of scalar are observed in the wake of the obstacles and lower amounts in front of the obstacles. This regular pattern is particularly evident in the regular cube array C10S compared to the random height array RM10S. From $z / h=0.3$ up to the mean canopy height, the area-average concentration values of the scalar in RM10S are less than C10S and the reverse is observed above the canopy top. This is due to the strong streamwise advection within the canopy and weaker advection above the canopy in RM10S.

The variations of dispersive and turbulent scalar fluxes with height have been obtained; this would be an extremely challenging task in field or laboratory experiments. These estimates are crucial in quantifying the scalar transport from different types of roughness surfaces. The surface flux of the scalar is found to be larger for the uniform height array compared to the random height array, which could be because of the higher turbulence intensities in the latter geometry. Also, relatively larger vertical flux is observed above the mean canopy height in RM10S. C10S exhibited a regular pattern of higher surface flux values in front of all the roughness elements near the area source whilst RM10S showed higher surface flux values mainly in front of the tallest obstacles. On the leeward side and adjacent to the block, because of secondary recirculation region, relatively higher flux values are observed compared to the wake region. It was found that around $z / h \approx 0.1$, the minimum advective fluxes occur around the tallest roughness elements, whereas the turbulent flux is more or less constant around all the blocks. Increasing surface flux contributions around blocks of increasing height are observed. Eddy diffusivities are estimated for the two geometries and it is observed that these values are negative at many locations within the mean canopy height. Standard RANS models would be quite unable to capture such regions, so concentrations elsewhere would also likely be inadequately predicted.

This study has clearly demonstrated that canopy ventilation is very much affected by the surface morphology and that large-eddy simulation techniques can be successfully used to explore such effects for surface scalar sources, provided near-wall resolution is sufficient to resolve adequately the concentration boundary layers on the surfaces. It is probable, nonetheless, that at full-scale (field) Reynolds numbers, when such boundary layers are relatively even thinner, parameterisation of the concentration (or thermal) processes occuring near boundaries will be required. It is anticipated that this can be achieved by using appropriate wall models for flow and/or scalar and this will form the subject of a future publication.

Acknowledgements This project is funded by the Natural Environment Research Council, through its National Centre for Atmospheric Sciences, Grant No. R8/H12/38. 


\section{References}

Barlow JF, Belcher SE (2004) Scalar fluxes from urban street canyons. Part I: Laboratory simulation. Boundary-Layer Meteorol 113:369-385

Cai XM, Barlow JF, Belcher SE (2008) Dispersion and transfer of passive scalars in and above street canyons-large-eddy simulations. Atmos Environ 42:5885-5895

Cheng H, Castro IP (2002) Near wall flow over urban-like roughness. BoundaryLayer Meteorol 104:229-259

Coceal O, Thomas TG, Castro IP, Belcher SE (2006) Mean flow and turbulence statistics over groups of urban-like cubical obstacles. Boundary-Layer Meteorol 121:491-519

Coceal O, Dobre A, Thomas TG, Belcher SE (2007) Structure of turbulent flow over regular arrays of cubical roughness. J Fluid Mech 589:375-409

Finnigan J (2000) Turbulence in plant canopies. Annu Rev Fluid Mech 32:519-571

Georgakis C, Santamouris M (2006) Experimental investigation of air flow and temperature distribution in deep urban canyons for natural ventilation purposes. Energy and Build 38:367-376

Grimmond CSB, Oke TR (2002) Turbulent heat fluxes in urban areas: observation and a local - scale urban meteorological parameterization scheme (LUMPS). J App Meteorol 41:792-810

Harman IN, Barlow JF, Belcher SE (2004) Scalar fluxes from urban street canyons. Part II: Model. Boundary-Layer Meteorol 113:387-409

Huang H, Ooka R, Kato S (2005) Urban thermal environment measurements and numerical simulation for an actual complex urban area covering a large district heating and cooling system in summer. Atmos Environ 39:6362-6375

Idczak M, Mestayer P, Rosant JM, Sini JF, Violleau M (2007) Micrometeorological measurements in a street canyon during the joint ATREUS-PICADA experiment. Boundary-Layer Meteorol 124:25-41

Ji Y, Lomas KJ, Cook MJ (2009) Hybrid ventilation for low energy building design in south China. Build and Environ 44:2245-2255

Kanda M (2006) Large-eddy simulations on the effects of surface geometry of building arrays on turbulent organized structures. Boundary-Layer Meteorol 118:151168

Kanda M, Moriizumi T (2009) Momentum and heat transfer over urban-like surfaces. Boundary-Layer Meteorol 131:385-401

Kanda M, Moriwaki R, Kasamatsu F (2004) Large-eddy simulation of turbulent organized structures within and above explicitly resolved cube arrays. Boundary-Layer Meteorol 112:343-368

Kim JJ, Baik JJ (1999) A numerical study of thermal effects on flow and pollutant dispersion in urban street canyons. J App Meterol 38:1249-1261

Kim JJ, Baik JJ (2001) Urban street-canyon flows with bottom heating. Atmos Environ 35:3395-3404

Kovar-Panskus A, Moulinneuf L, Savory E, Abdelqari A, Sini JF, Rosant JM, Robins A, Toy N (2002) A wind tunnel investigation of the influence of solar-induced wall-heating on the flow regime within a simulated urban street canyon. Water Air Soil Pollut 2:555-571 
Louka P, Vachon G, Sini JF, Mestayer PG, Rosant JM (2002) Thermal effects on the airflow in a street canyon-Nantes'99 experimental results and model simulations. Water Air Soil Pollut 2:351-364

Mathey M, Fröhlich J, Rodi W (1999) LES of heat transfer in turbulent flow over a wall-mounted matrix of cubes. In: Voke PR, Sandham ND, Kleiser L (eds) Direct and Large Eddy Simulation III (ERCOFTAC series), Kluwer, Dordrecht, pp 51-62

Meinders ER, Hanjalić K (1999) Vortex structure and heat transfer in turbulent flow over a wall-mounted matrix of cubes. Int J Heat and Fluid Flow 20:255-267

Offerle O, Eliasson I, Grimmond CSB, Holmer B (2007) Surface heating in relation to air temperature, wind and turbulence in an urban street canyon. Boundary-Layer Meteorol 122:273-292

Pascheke F, Barlow JF, Robins A (2008) Wind-tunnel modelling of dispersion from a scalar area source in urban-like roughness. Boundary-Layer Meteorol 126:103124

Sini JF, Anquetin S, Mestayer PG (1996) Pollutant dispersion and thermal effects in urban street canyons. Atmos Environ 30:2659-2677

Solazzo E, Britter RE (2007) Transfer processes in a simulated urban street canyon. Boundary-Layer Meteorol 124:43-60

STAR-CD (2008) CD-Adapco's CCM user guide, STAR-CD Version 4.08

Xie ZT, Castro IP (2006) LES and RANS for turbulent flow over arrays of wallmounted obstacles. Flow Turbulence and Combust 76:291-392

Xie ZT, Castro IP (2009) Large-eddy simulation for flow and dispersion in urban streets. Atmos Environ 43:2174-2185

Xie ZT, Hayden P, Voke PR, Robins AG (2004) Large-eddy simulation of dispersion: comparison between elevated and ground-level sources. J Turbulence 5:1-16

Xie ZT, Coceal O, Castro IP (2008) Large-eddy simulation of flows over random urban-like obstacles. Boundary-Layer Meteorol 129:1-23

Yang Y, Shao Y (2008) Numerical simulations of flow and pollution dispersion in urban atmospheric boundary layers. Environ Model Softw 23:906-921 